\title{
Peer To Peer Approach based Replica AND LOCALITY AWARENESS TO MANAGE AND DisSEMINATE DATA IN VEHICULAR AD HOC NETWORKS
}

\author{
Abdelkader Guezzi ${ }^{1}$, Abderrahmane Lakas $^{2}$, Ahmed Korichi $^{1}$, Sarra Cherbal ${ }^{3}$ \\ ${ }^{1}$ Computer science and information technology department, \\ Kasdi Merbah University, Ouargla, Algeria \\ ${ }^{2}$ College of Information Technology UAE University, Al Ain, UAE \\ ${ }^{3}$ University of Ferhat Abbas Setif1 19000 Setif, Algeria
}

\begin{abstract}
Distributed Hash Table (DHT) based structured peer-to-peer (P2P) systems provide an efficient method of disseminating information in a VANET environment owing to its high performance and properties (e.g., self-organization, decentralization, scalability, etc.). The topology of ad hoc vehicle networks (VANET) varies dynamically; its disconnections are frequent due to the high movement of vehicles. In such a topology, information availability is an ultimate problem for vehicles, in general, connect and disconnect frequently from the network. Data replication is an appropriate and adequate solution to this problem. In this contribution, to increase the accessibility of data, which also increases the success rate of the lookup, a method based on replication in the Vanet network is proposed named LAaR-Vanet. Also, this replication strategy is combined with a locality-awareness method to promote the same purpose and to avoid the problems of long paths. The performance of the proposed solution is assessed by a series of in-depth simulations in urban areas. The obtained results indicate the efficiency of the proposed approach, in terms of the following metrics: lookup success rate, the delay, and the number of the logical hop.
\end{abstract}

\section{KEYWORDS}

Vehicular Ad-hoc Network (VANET), Structured P2P Systems, Distributed Hash Table (DHT), Locality Awareness, Replication.

\section{INTRODUCTION}

A VANET is an infrastructure-less wireless network that allows vehicle connectivity and information sharing for safe driving. As a result, VANET networks are designed for delivering essential services related to safety, traffic management, and driving amenities, such as infotainment and internet connectivity [1].

The main difference between simple Mobile Ad hoc NETworks (MANET) and VANET is attributed to the latter's vehicle motion and their known trajectories through time. This is why they are more controllable. Yet, designing an efficient information dissemination protocol in VANET is challenging owing to the instability vis-à-vis nod churn of such kind of network at any given time.

Moreover, structured peer to peer (P2P) overlays, built on the Distributed Hash Table (DHT), demonstrate high routing and self-organization capabilities in the VANET environment. The 
implementation of structured $\mathrm{P} 2 \mathrm{P}$ networks exploits a consistent hash function to assign unique identifiers (IDs) to the participating nodes (and the resources they share) from a universal identifier space.

There is a predefined function that maps resource IDs to a single peer, peers responsible for storing these resources, and/or their references. Hence, to search for any resource, the requesting nod has to find the route until the responsible nod contains the desired resource. For this, every nod keeps some state (routing table) about certain peers to find the route of search requests. Examples of structured peer to peer systems are Chord [2], Pastry [3], and so forth.

The VANET structure has very dynamic behavior. Peers may join or leave the network at any time, which greatly reduces data availability. To cope with this problem, a data replication mechanism, which consists of replicating the data in other nodes instead of one node, is proposed (LAaR-Vanet). Such a paradigm is fundamental to increase data availability and enhance load balancing, as it can reduce the lookup path.

Since the definition of IDs in a structured P2P network is random, nodes are distributed in the overlay without considering their real physical positions. So nodes neighboring in the overlay can situate very far in the real system (Figure 1). This mismatch results in redundant traffic and high end-to-end latency. To take into account the physical positions of nodes, a modification in the definition of IDs in the Chord protocol on VANET is proposed. This solution is called locality awareness.

The results of the simulation demonstrate the efficiency and the scalability of LAaR-Vanet in several settings. The rest of the paper is organized as follows. Section 2 provides a brief description of background concepts such as structured P2P system, locality awareness, and replication. Section 3 reviews the related work and provides an overview of the existing approach. Section 4 is rather a focus on the proposed model. Section 5 details the performance evaluation of LAaR-Vanet through an extensive simulation study. Section 6 is devoted to the conclusion of the paper and to the coming research directions outline. 


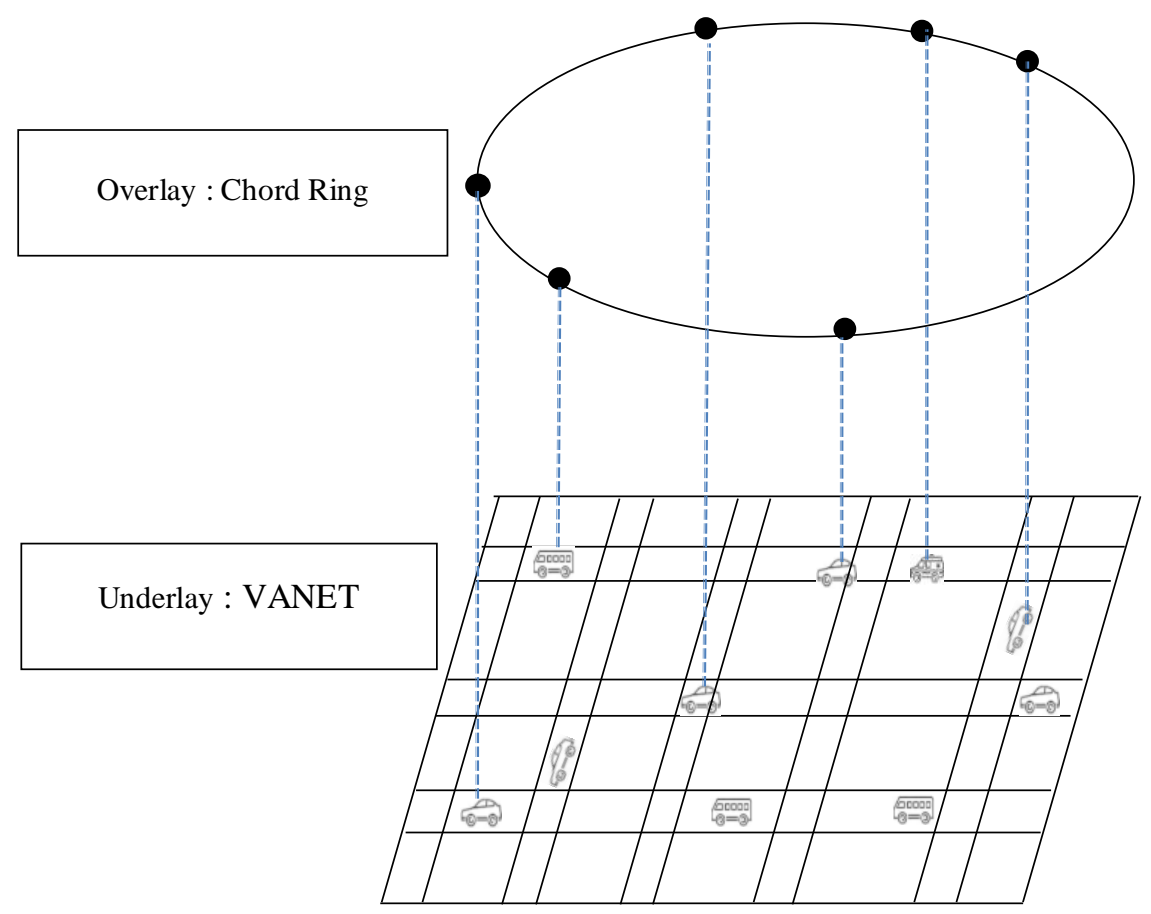

Figure 1. DHT of Vehicles

\section{BACKGROUND CONCEPTS}

\subsection{Structured P2P Overlay}

Structured peer- to -peer overlays network creates a virtual controlled topology above the basic transport layer [4]. These overlays utilize Distributed Hash Tables (DHTs) and offer various features like robust wide-area routing architectures, an efficient search of data items, storage, and self-organization.

Chord [2] is a structured peer to peer protocol mostly used. It offers well support for locating a peer that stocks desired content. In chord, every node and content object are assigned by m-bit identifier using a consistent hashing strategy (SHA-1) exploited in Chord protocol. Generally, Chord is deployed on the application layer as a P2P overlay and acts elegantly owing to the simplicity of the design and scalability to construct large-scale overlay networks without high complexity. In an $\mathrm{N}$-node Chord overlay, each node has to and can, respectively, keep contact with only and perform query within both $\mathrm{O}(\log \mathrm{N})$ nodes \& steps.

Moreover, to retain the stability of Chord, all nodes have to maintain some local information (the predecessor and the successor) to keep the consistency of Chord.

In Chord, the space of identifiers can be seen as an identifiers circle where identifiers are denoted from 0 to $2^{m-1}$, such as $m$ is the number of bits in the identifier. This is what is called a Chord ring. In this identifier ring, all peers have to maintain links to its predecessor, and successor, through this last, all lookup requests are conducted around the ring. When the peer that contains the desired data is found, the lookup process is terminated. 
Each node keeps other routing information to enhance routing performance, this information making what is called the finger table. This latter is a routing table contains $\mathrm{m}$ line as a maximum. The $i^{\text {th }}$ line in the finger table of any node $\mathrm{n}$ refers to the node that follows $n$ by, at least, $2^{i-l}$ in the ring where $1 \leq i \leq m$, and each operation is modulo $2^{m}$.

The entries of the finger table are updated accordingly through a cooperative stabilization procedure executed at each peer. This is essential for keeping the stability of the ring. To minimize the influence of churn (peer leaving and joining), each peer has to update the routing table through a periodical mutual exchange of messages. The maintenance cost is proportional to the churn rate: the more the churn rate is high, the shorter the interval of periodic maintenance is, and the higher the maintenance cost is.

\subsection{Replication}

In a structured peer to peer system, each object is identified by the hashing of its name to obtain the object's key. This object is destined to be stored in one node which has the closest identifier to the key. This node is the so-called either root node or master node. Replication consists of replicating this data in more than one node; replication allows increasing for instance data availability, decreasing response time, and so on.

\subsection{Locality Awareness}

As mentioned before in structured peer- to -peer overlay, the dispensation of keys to the node does not consider physical topology. In this case, two nodes could be found physically adjacent. Yet, in the overlay, they are very far from each other; this mismatch problem makes the length of the routing path very long. This increases the end to end delay.

Locality-awareness is proposed as an answer to this problem between the physical network and its overlay. In other words, at the overlay building stage, the physical network topology is factored into consideration.

The locality awareness allows having neighbor nodes in the overlay, which are as well so close in the underlay. This state provides a faster and more efficient search.

To achieve the Locality-awareness approach, several methods may be used to build the overlay. Example: Broadcasting, clustering, and node-ID assignment [5]. Locality-awareness can accelerate the lookup process, improve the success rate, and so on.

\section{RELATED WORKS}

\subsection{Locality Awareness}

In [6], the authors presented a traffic information system (Peertis) based on a structured peer to peer system; Peertis allows data sharing between vehicles, which form the overlay structure, owing to internet connections.

The authors use CAN as a peer to peer protocol. In their approach, roads are grouped into road sectors; each with a unique ID that is used as a key in the DHT; each sector has a vehicle responsible for all the data related to this section. The authors propose to keep the neighboring sections of roads close in the resulted overlay; this idea can be reached by replacing the hashing function with neighboring coordinates in the underlying topology. 
In [7], they propose a location-aware Chord in wireless environments (WILCO). On the one hand, in WILCO, peers create the logical structure with their predecessor and successor using their ID based on geographical location information to achieve the high quality of service in wireless networks. On the other hand, modification in the finger table can improve the new overlay resulted based on location-aware. However, this approach is disturbed by the mobility of peers, which causes many variations of geographical location; the latter decreases the network scalability.

MANET Chord GNP, which is proposed in [8], is based on the building of the chord-overlay considering the physical topology. GNP (global network positioning) system is used to assign Peer-ID according to the peer's geometric-coordinates. A group of nodes is chosen as landmarks to help other nodes to participate in the network. If the landmark-node moves or disconnect from the network, the nodes, which has the closest logical ID will replace it. A unique temporary ID is assigned to each node to define its location in the overlay. The lookup and the resource placement procedure are similar to the basic Chord.

Many works of locality awareness in VANET based on the clustering paradigm are proposed. Clustering is a method of grouping vehicles considering some factors such as direction, speed, and physical location of vehicles. In [9], for example, CBLR (cluster-based location routing) is proposed; vehicles share their states using HELLO messages. This protocol CBLR is enhanced in [10]. In [11], the authors propose to form clusters of vehicles based on their velocity.

For more information about locality awareness in the mobile network, the reader has to refer to [5].

Because VANET's very dynamic topology, few studies have been conducted on its environment. Yet, the proposed locality awareness in VANET is combined with a replica approach. Moreover, the mechanism proposed for building the overlay allows forming a strong overlay network.

\subsection{Replica}

In [12], the authors propose V-PADA (vehicle-platoon-aware data) for VANETs. In V-PADA, the vehicles are gathered; they form together a platoon; cars in a platoon are requested to contribute a portion of their buffers for saving a copy of data for other cars of the same platoon and also share data with them. When a vehicle disconnect from the platoon, it transfers interesting data to other cars of the same platoon. So, data is still accessible by the other vehicles. The drawback of this method is that frequent vehicles' leaving and their entering into the platoon may incur additional computation overhead.

In [13], an approach is proposed where data is replicated in Road Side Unit (RSU) to offer rapid information delivery and therefore improve the data availability in VANETs. In this method, the data access pattern and driving pattern are used to select the data item which should be replicated in the RSU. The replicated data in the RSUs are requested to send directly to the vehicle without having communication with RSUs in which the original data is available. The main limitation of this method is the data replication process which may take too much time if the size of the data is large; also their approach is limited only in the environment which contains RSU.

To obtain a high data availability in VANET, the authors of [14] propose a scheme based replica. In this approach, each vehicle stops in a parking lot has to replicate data between other vehicles of the same parking lot during its stop, and if it moves to other parking lots, it replicates, also, data with other vehicles. 
In [15], the capabilities of all nodes in terms of processing or storage are taken into account. So, the authors suggest a replication method based on a distributed randomized algorithm in which the total number of data replica disseminated in the network is controlled. In the authors' proposed method, the number of data replicated is limited, and every vehicle is assigned with such a value that indicates the quantity of data replicas disseminated by the vehicles. Although the author's approach seems feasible, it is assumed that the approach's conditions are unrealizable in the actual environment.

In [16], a routing protocol so-called CCR in VANET is proposed. It is a capacity-constrained replication approach for data dissemination in VANET where each vehicle adapts its replication maximum according to the varying network capacity. Although CCR reaches a high delivery success ratio, it leads to more overhead in the network.

In [17], a distributed data replication method called EDDA has been proposed. The main idea in EDDA is to let the node holder disseminate the data to multiple nodes to speed up the dissemination process, and then to calculate the number of communication steps for the network to enter into a stable status.

\section{Proposal}

\subsection{Assumed Scenario}

In the proposed approach, a VANET in the urban environment is pretended. In this environment, all vehicles are equipped with a wireless network interface based IEEE 802.11p with a fixed range through which these vehicles can connect with each other. The IEEE $802.11 \mathrm{p}$ is wellknown as dedicated short-range communication (DSRC) [18] and is an approved improvement to the IEEE 802.11 standard to add wireless access in VANET.

Using Beacon, which is a short-range single-hop periodic message broadcast, all vehicles are supposed to share information about themselves concerning speed, position, and direction. In fact, all this information is borne by Beacon.

In LAaR-Vanet, Chord protocol is used because it is a simple protocol, high performance, and provable correctness.

However, LAaR-Vanet can be applicable to any of DHT systems like Pastry [3] and CAN [19] as their overlays use ID-assignment to place the nodes.

\subsection{Replication}

In Chord DHT [2], every node conserves other routing information which is named the fingertable. The latter is a routing table which contains $m$ lines (for $m$-bit identifier space) as a maximum. The $i^{\text {th }}$ line in the finger-table of any node $n$ contains the identifier of a node that succeeds $n$ by at least $2^{i-1}$ on the identifier circle, where $1 \leq i \leq m$ and all operations are modulo $2^{m}$.

To benefit from the robustness of the query routing mechanism used in Chord, especially between the node and its finger, one proposes to replicate data in the finger node list of the owner node, aiming therefore to make an answer to a data-loss problem caused by node-churn. Indeed, a new data replication strategy called finger node replication has been proposed called LAaRVanet. 
In LAaR-Vanet, the data is replicated into a set of finger nodes of the master node. Doing so, the probability to find a replica node increases in this kind of network under the continuous change of the overlay. LAaR-Vanet allows increasing data availability in the system and obtaining a high lookup success rate. This replica method can, as well, reduce the number of hops required to localize the requested data.

The process of replicating data is summarized in the following steps:

For every received resource,

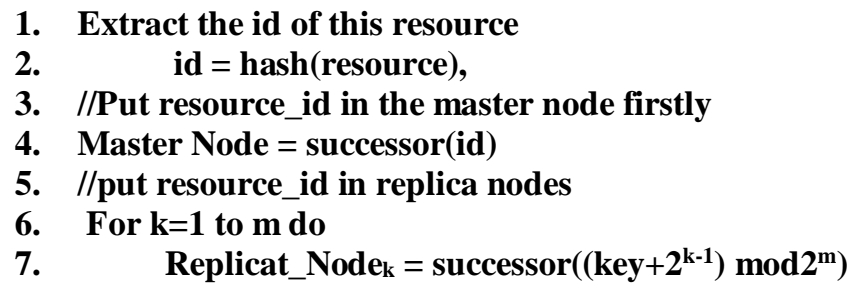

The Vanet topology is very affected by the joining/leave of nodes. So, the stabilization mechanism can deal with this situation. To avoid the generation of more overhead in the overlay by the proposed replica approach (LAaR-Vanet), replicating data in the stabilization step is suggested. Consequently, when a node updates the list of its finger node, it replicates the data in these nodes immediately.

In the lookup procedure, the node starts by checking whether the node is responsible or a replica for the requested data. In this case, the request is solved. If the node is neither the holder nor the replica, then it transmits the request to the node that has the largest ID in the finger table, but inferior or equal to the requested key.

For example, in Figure 2, the responsible node of key=3 is 4 . The latter has nodes 8 and 12 as finger nodes, so key 3 also replicated in these nodes $(8,12)$.

Node 8 that looks for key=3; in this case node 8 contains key 3 as a replica node, so it does not transmit the request.

As shown in Figure 2, in basic Chord, the lookup request is passed from node 8 to node 0 and then to node 4 . In contrast, in the replication method, node 8 contains the desired object, which presents a more reduced lookup path and a decreasing hops number after using LAaR-Vanet. However, reducing the logical hop number also involves reducing the physical hop number. 


\begin{tabular}{|c|c|}
\hline $\mathrm{K}=$ & node \\
\hline 1 & 1 \\
\hline 2 & 2 \\
\hline 3 & 4 \\
\hline 4 & 8 \\
\hline
\end{tabular}

FT of 0

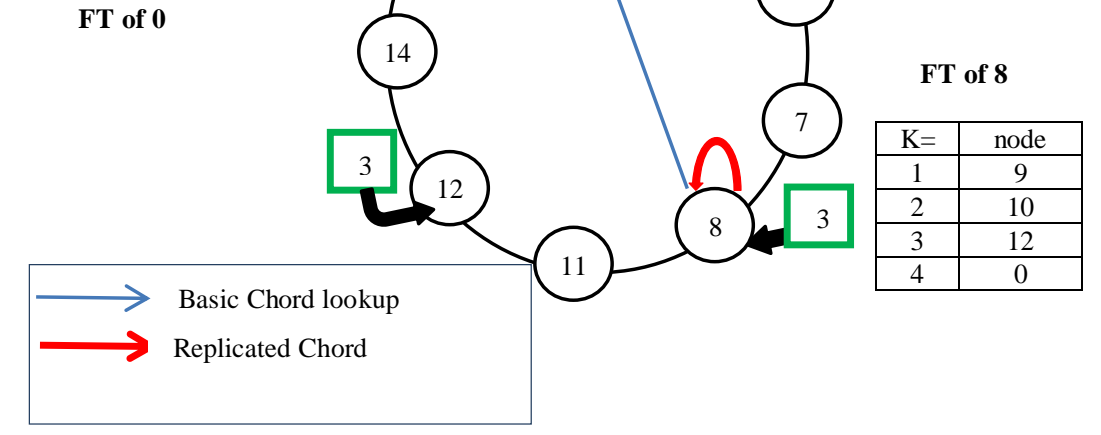

Figure 2. A lookup path example, before and after using the replication method

\subsection{Locality Awareness}

In Chord overlay, nodes obtain their IDs in a random manner, such as hashing their IP address; this method does not consider the physical position of the node which causes no correspondence between physical neighbors and overlay neighbors. The solution to this mismatch problem is called locality awareness, which takes into consideration the physical position in building the overlay. The obtained result from the application of locality awareness is an overlay neighbor in correspondence with their physical neighborhood. As it is presented in Figure 3, nodes that are neighbors in the physical underlay have closest IDs and they are placed as neighbors in the logical overlay.

This latter is called ID-assignment which is based on locality awareness. Therefore, in LAaRVanet, the aim is to change the ID definition by considering the physical neighborhood and to adapt it to the VANET environment.

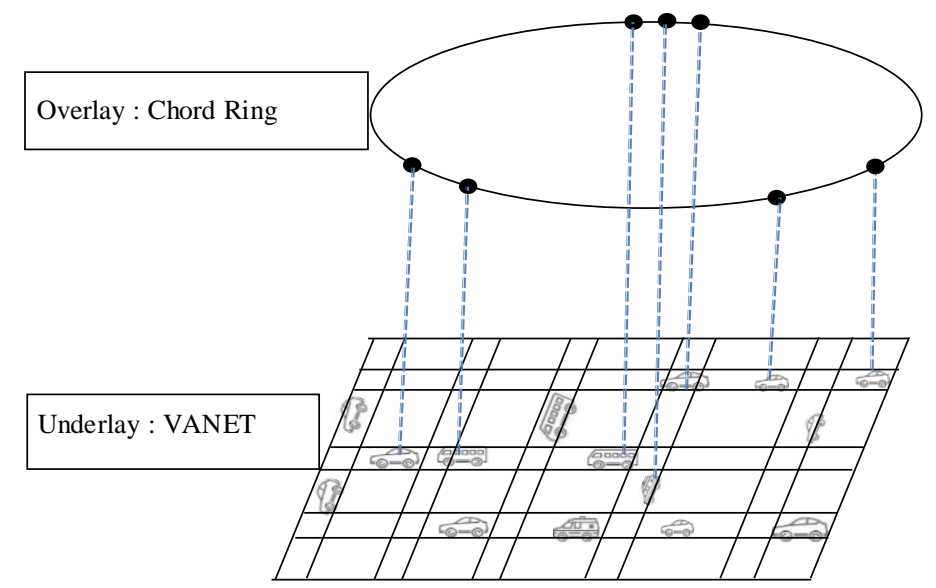

Figure 3. Locality-awareness applied on Chord over VANET

The most stable vehicles in the route are chosen to act as Bootstrap Vehicle (BV). So, public transport like city-bus and taxi-service selected to act as a bootstrap vehicle for the overlay, using 
International Journal of Computer Networks \& Communications (IJCNC) Vol.12, No.6, November 2020

their mac-address in the Vehicle-ID calculation process. It is also assumed that only BV can reply to join call requests.

Separately, all BVs calculate their hash-id according to this equation:

BV_ID $=$ hash $\left(\operatorname{mac}\right.$ address of BV)mod $2^{\mathrm{k}}+$ hash(ip address of BV)mod $2^{\mathrm{m}-\mathrm{k}} \quad(+$ denote the concatenation)

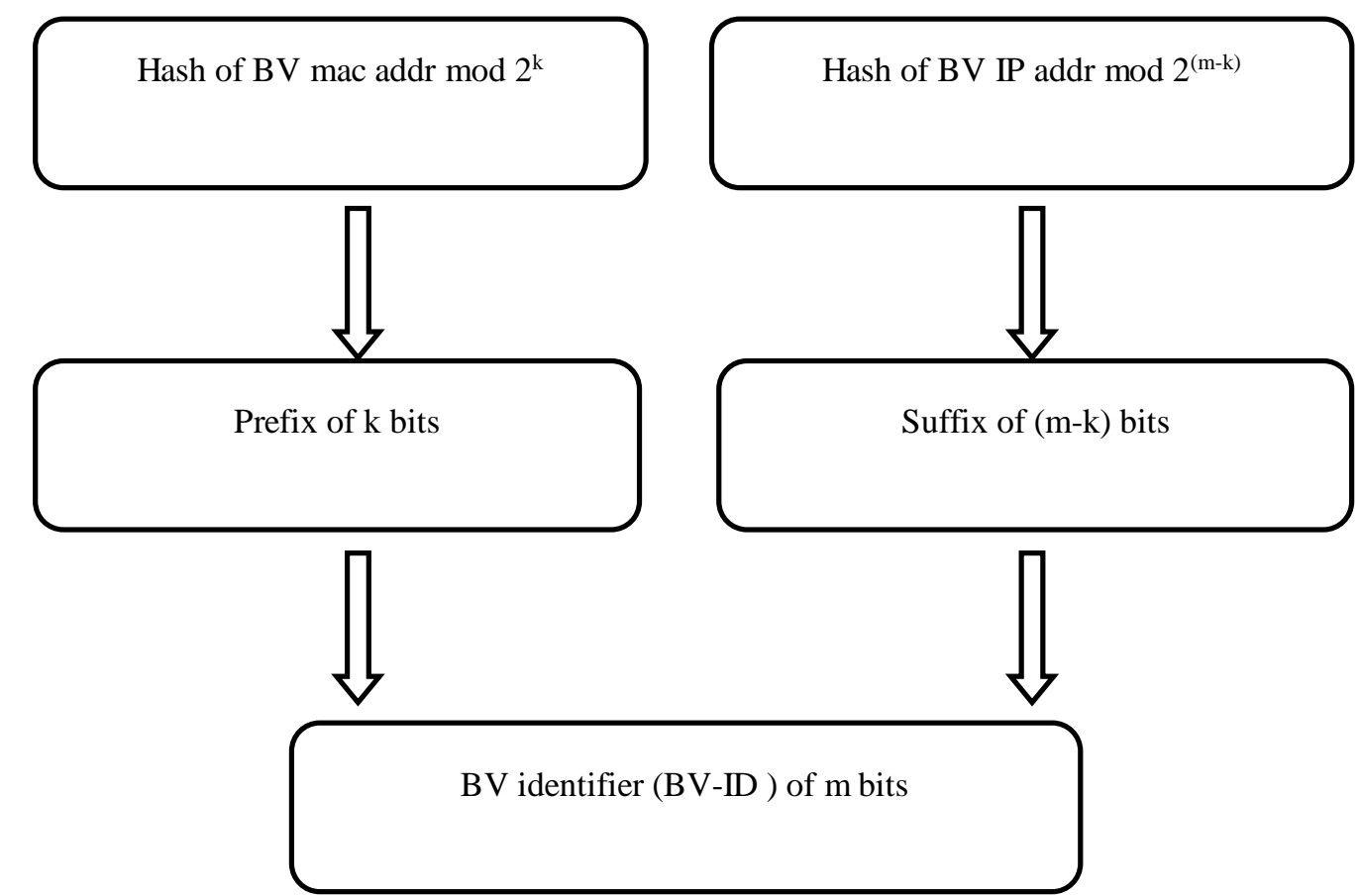

Figure 4. The calculation of BV identifier (BV-ID)

Moreover, all ordinary vehicles calculate their reference according to this equation based on the reference of the Best BV.

The Best Bootstrap Vehicle (BBV) has been chosen according to its velocity and direction. In this manner, the lifetime of the link between a vehicle and its BBV can be prolonged as long as possible.

All vehicles calculate their hash-id according to this equation:

Vehicle_ID $=$ hash $(\operatorname{mac}$ address of BBV $) \bmod 2^{\mathrm{k}}+$ hash(ip address of Node $) \bmod 2^{\mathrm{m}-\mathrm{k}} \quad(+$ denote the concatenation) 


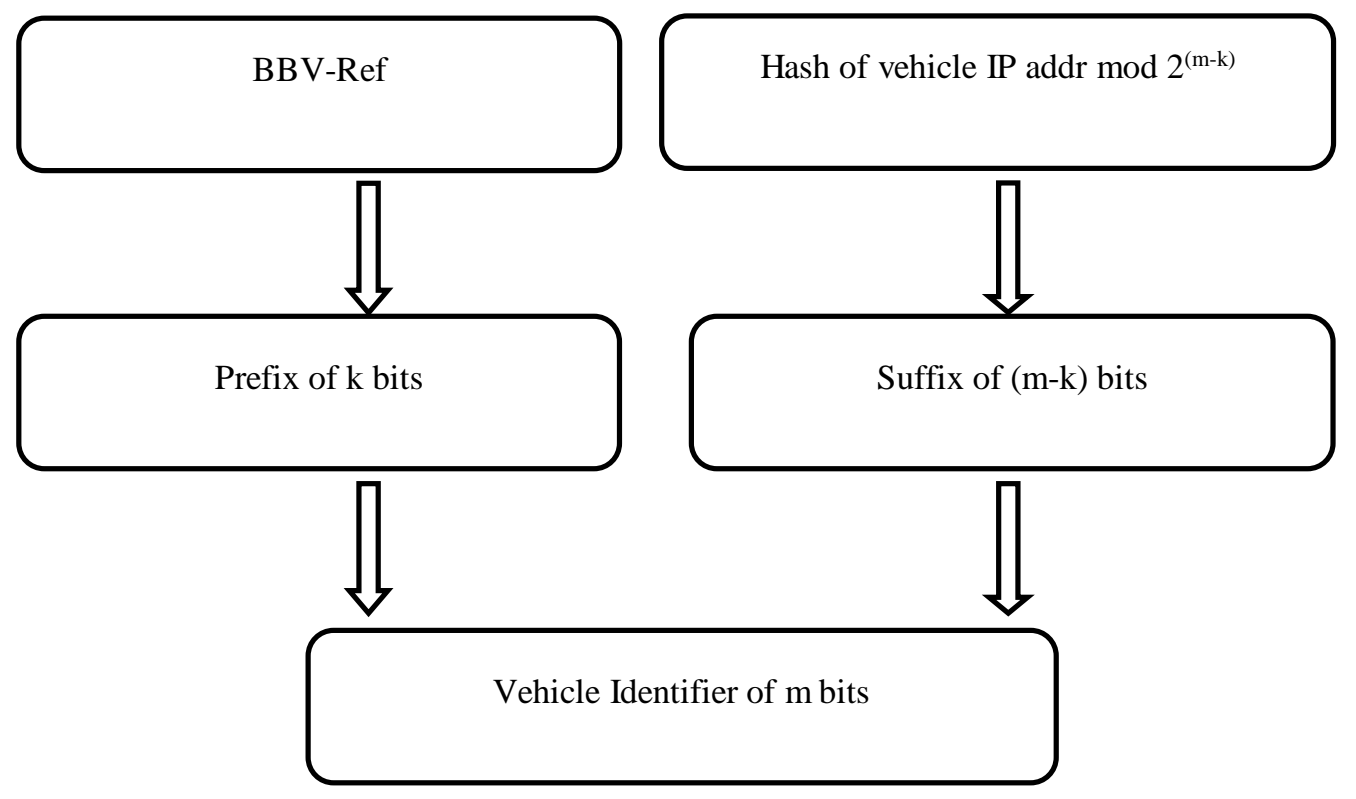

Figure 5. The calculation of Vehicle Identifier

The joined procedure of vehicle summarized in the following steps:

8. Newly arriving vehicle (Vnew) sends a join request to 1-hop neighboring.

9. All 1-hop neighboring B Vexist reply to the request with the details of an existing BVexist

10. Vnew choose the best $B V(B B V)$ according to defined information

11. Vnew calculate Vid( hash-id) based on the chosen BBV
a. $\quad$ Prefix $=$ hash mac address of BBV
b. $\quad$ Suffix $=$ hash ip address of Vnew
c. $\quad$ Node ID = Prefix + Suffix
(+ denote concatenation)

12. Updating finger tables, successor links, and predecessor links of existing overlay, affected by joining of Vnew,

13. Transfer of content objects to Vnew from their successor

In LAaR-Vanet, after Vnew calculates its hash-id, the remaining process is handled by DHT overlay maintenance procedures. Every node in DHT executes a stabilization procedure periodically. In this procedure, the node checks its successor and examines about its predecessor. In this manner, if any node enters in the system, it is discovered in a predefined period. Besides, every node performs a fix-finger procedure from time to time to refreshes its finger-table. The details of the fix-finger and stabilization process subsist in [2].

\subsubsection{BBV Departure or Displacement}

If the BBV leaves the network, all nodes related to it have to detect a new BBV, and then rebuild the overlay. So all vehicles will have a new id calculated according to the Ref of this new BBV. Additionally, if BBV moves, it does not have to change its ID unless its IP address is changed.

\subsubsection{Vehicle's Departure or Displacement}

If the vehicle's departure is predictable, the vehicle sends its resource to its successor in the overlay and also notifies its predecessor to update the finger table and successor list. But, if the 
departure is unpredictable, the overlay will discover it is leaving in the coming step of stabilization.

In the case of the vehicle displacement, if the vehicle's BBV is not more accessible, the vehicle has to update its ID according to the new BBV. This can happen when BBV reaches its last stop of the route or gets halted due to any other reason for more than a threshold time limit.

\section{Simulation EnVironment And Results}

We have simulated LAaR-Vanet by using Oversim [20] over OMNeT++ [21] and Traffic control Interface (TraCI)[22] client for OMNeT++/MiXiM [23] framework. OMNeT++ is an opensource, component-based, discrete event simulation environment coded in C++. OverSim is an open-source simulation framework designed for peer to peer overlay networks to be used over OMNeT++ simulation environment.

Oversim hold several modules for structured peer -to- peer systems e.g. Chord, Kademlia, and Pastry. We have used its Chord module for simulating DHT of vehicles. MOVE (Mobility model generator for VEhicular networks) [24] is used to generate realistic Vehicle movements. MOVE is built on top of micro traffic simulator SUMO (Simulation of Urban MObility) [25].

OMNET++ provides the implementation of the IEEE $802.11 \mathrm{p}$ standard which is the suitable protocol for the vehicular environment. The vehicle's movement is simulated by a GUI offered by the MOVE simulator. With this GUI, the speed, departure time, of vehicles, their routes, and so forth can be represented simply to simulate the mobility of vehicles. TraCI offers a TCP built on the client-server architecture. MOVE/SUMO acts as TraCI server whereas OMNET++/INET acts as TraCI client to exchange commands using TCP connections between them.

The performance of LAaR-Vanet is compared against that of the original mobile Chord algorithm, based on the following performance metrics:

\subsection{Lookup Hop Count}

This is the average hop count or vehicles browsed of every successful lookup in the overlay. Logical Hop counts reflect the lookup speed which is an important metric in the VANET environment. The average hop number is given by:

$$
O=\left(\sum_{k}^{r} H_{k}\right) / R
$$

Such as $R$ is the total number of lookup requests and $H_{k}$ is the number of hops browsed by each lookup.

\subsection{Lookup Delay Time}

This is the average time required to deliver a response to the intended requester. The average lookup latency or file discovery delay is given by:

$$
D=\left(\sum_{k}^{l} T_{k}\right) / L
$$


Such that $L$ is the total number of lookup requests, and $T_{k}$ is the delay elapsed by 1 lookup request from the source to the target node.

\subsection{Lookup Success Rate}

This is the ratio of the number of lookups resolved to the number of all resource lookups. The Success ratio is done by the following expression:

$$
E=R / L \times 100
$$

Such as $L$ is the total number of lookup requests and $R$ is the number of resolved lookup requests. The simulation parameters are summarized in Table 1.

Table 1: Simulation Parameters

\begin{tabular}{|l|l|}
\hline Traffic type & $100,200,300,400,500$ \\
\hline Number of vehicles & $65 \mathrm{~km} /$ hour \\
\hline Vehicle properties & $45 \mathrm{~km} /$ hour \\
\hline $\begin{array}{l}\text { Maximum speed of } \\
\text { vehicles }\end{array}$ & $20 \%$ of total vehicles \\
\hline Maximum speed of BV & $35 \%$ of total vehicles \\
\hline Number of BV & $\begin{array}{l}\text { Maximum number of } \\
\text { requester at a time }\end{array}$ \\
\hline Ad hoc communication properties \\
\hline Protocol used & $802.11 \mathrm{p}$ \\
\hline $\begin{array}{l}\text { Transmission range of } \\
\text { vehicles }\end{array}$ & 200 meters \\
\hline DHT communication properties \\
\hline Protocol used & Chord \\
\hline Underlying protocol & TCP/IP \\
\hline Fix-finger period & $3 \mathrm{~s}$ \\
\hline Stabilization period & $3 \mathrm{~s}$ \\
\hline
\end{tabular}

\subsection{Simulation Results}

\subsubsection{Lookup Success Rate}

We study the lookup success rate when the number of vehicles varies from 100 to 500 . The results are clarified in Figure. 6. In Figure 6, it can be observed that the lookup success rate generated by LAaR-Vanet is higher than the derived from the mobile Chord. And they decrease as soon as the vehicle's number increase, which is normal, these results prove the efficiency of the proposed method (LAaR-Vanet) in term of data availability. 
International Journal of Computer Networks \& Communications (IJCNC) Vol.12, No.6, November 2020

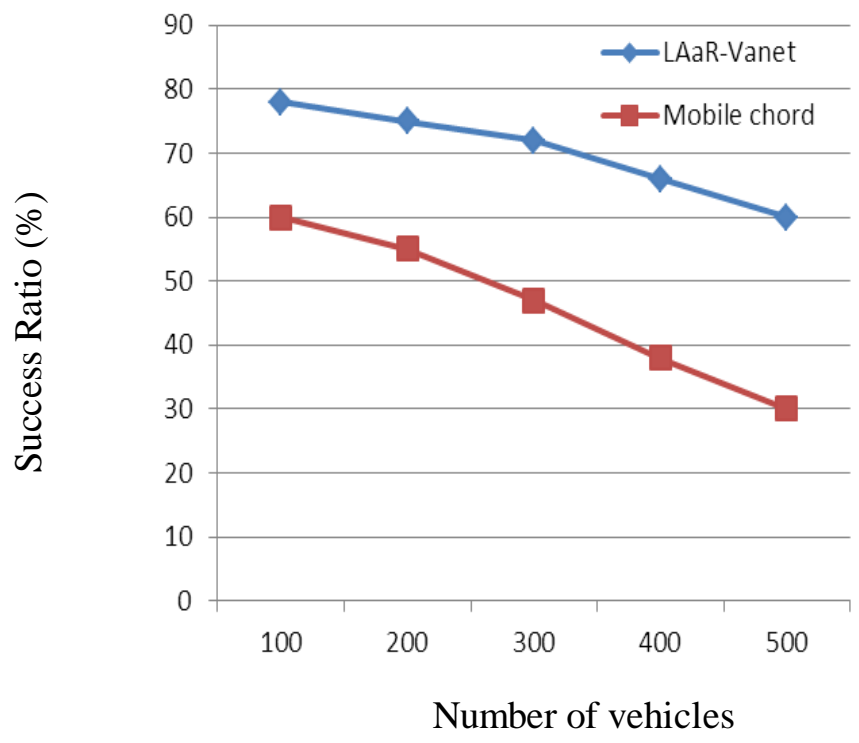

Fig 6. Lookup success rate: LAaR-Vanet versus mobile chord

\subsubsection{Lookup Hop Count}

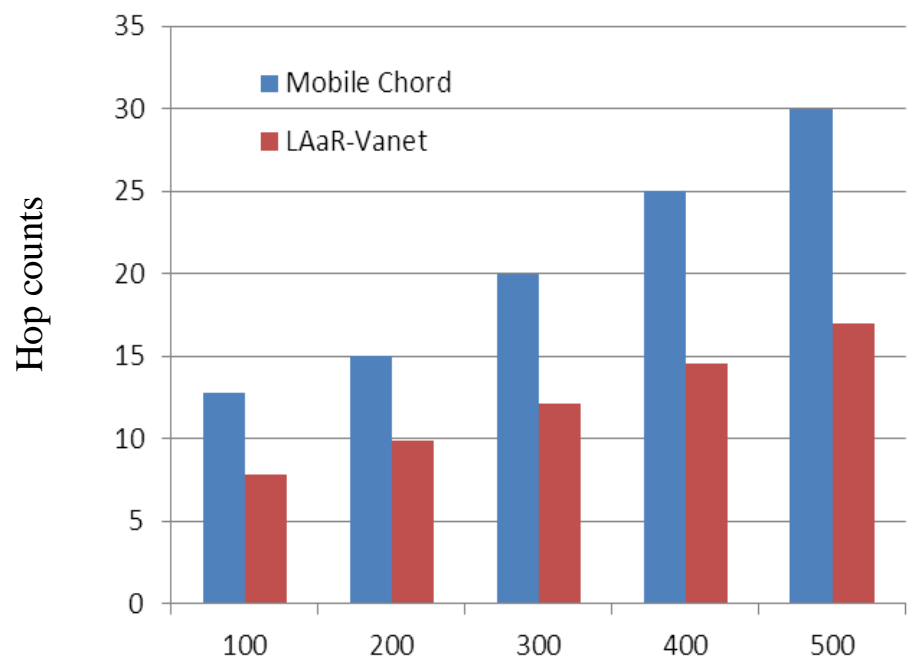

Number of vehicles

Fig 7. Lookup hop count: LAaR-Vanet versus mobile chord

The main objective of locality awareness is to take underlay topology into consideration, so we study the lookup hop count in LAaR-Vanet against mobile chord when the network size varies from 100 to 500 vehicles. Figure 7 shows that the number of averages hops browsed in the proposed approach lower compared with the mobile Chord. And it still increases with the augmentation of network size, which is reasonable with more peers to browse by lookup requests. 


\subsubsection{Lookup Delay Time}

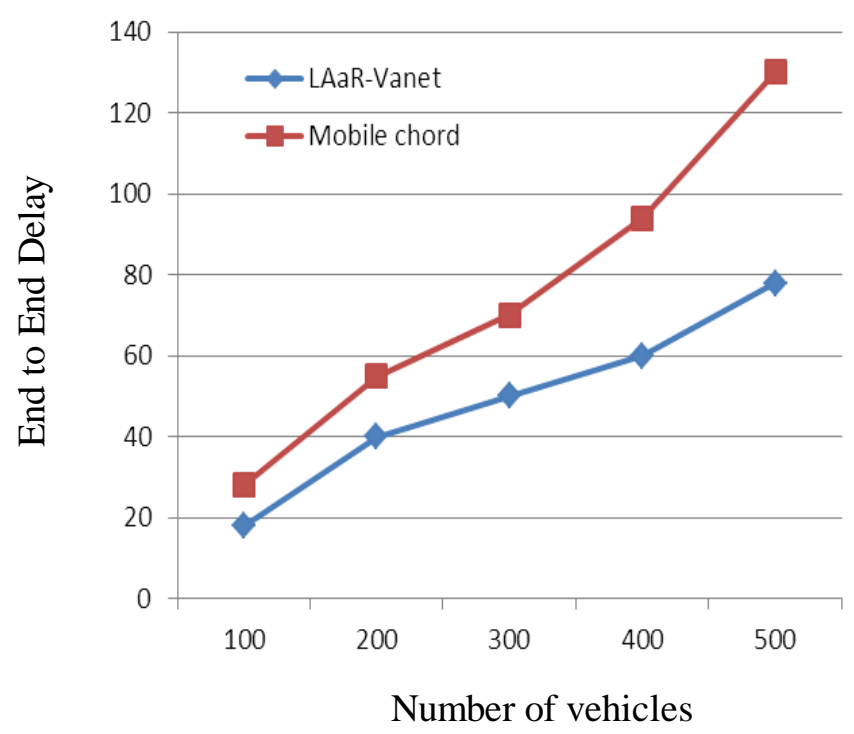

Figure 8. Lookup delay time: LAaR-Vanet versus mobile chord

We produce several lookup scenarios with the number of nodes increased from 100 to 500 vehicles. Simulation results demonstrated in Figure 8, Figure 8 shows that our method (LAaRVanet) performs much better compared with the mobile chord: Latencies in LAaR-Vanet are between 18 and 78 second compared to 28 and 130 second in the mobile Chord, and this is, on the one side, due to data availability in the closest holder, and on the other side, because in the building of the overlay, the physical topology is taken into consideration. This fact reduces the end- to -end delay. The reader can see the margin between the lookup latency of mobile chord and LAaR-Vanet getting larger the more the number of vehicles increases.

In this section, we show promising results of LAaR-Vanet. However, we observed during the simulation of the proposed approach a weakness in the area where the number of city buses is decreased or not available.

\section{CONCLUSION}

We have presented LAaR-Vanet, a replica method combined with an architecture based on a locality-awareness approach over structured P2P overlay for data dissemination in the VANET environment. The purpose of this method is to address the issue of data availability, also to reduce the number of hops browsed for each lookup and its delay. On one side, the replica method consists of replicating data in the finger node of Chord protocol, on the other side, locality-awareness is achieved by modifying the ID definition of Chord by considering the physical topology of vehicles.

Simulation's results show the effectiveness of the hybrid proposed approach (LAaR-Vanet). Results show a reduction in the number of lookup logical hops, the increase of lookup success ratio, and a decrease in the end to end delay. The performance is done in comparison with the mobile Chord. 
In our future work, our purpose is to achieve extended simulation results of LAaR-Vanet compared to other existing approaches, but not just in the urban environment. Also, we plan to implement the proposals in a practical real-world situation.

\section{CONFLICTS OF INTEREST}

The authors declare no conflict of interest.

\section{REFERENCES}

[1] G. Karagiannis et al., (2011) "Vehicular Networking: A Survey and Tutorial on Requirements, Architectures, Challenges, Standards and Solutions," IEEE Commun. Surv. Tutorials, vol. 13, no. 4, pp. 584-616, doi: 10.1109/SURV.2011.061411.00019.

[2] I. Stoica et al., (2003) "Chord: a scalable peer-to-peer lookup protocol for internet applications," IEEE/ACM Transactions on Networking, vol. 11, no. 1, pp. 17-32, doi: 10.1109/TNET.2002.808407.

[3] A. Rowstron and P. Druschel, (2001) "Pastry: Scalable, Decentralized Object Location, and Routing for Large-Scale Peer-to-Peer Systems,” in Middleware 2001, vol. 2218, R. Guerraoui, Ed. Berlin, Heidelberg: Springer Berlin Heidelberg, pp. 329-350.

[4] T. Pandey, D. Garg, and M. M. Gore, (2012) "Publish/subscribe based information dissemination over VANET utilizing DHT,” Front. Comput. Sci., doi: 10.1007/s11704-012-1154-7.

[5] S. Cherbal, A. Boukerram, and A. Boubetra, (2015) "A survey of locality-awareness solutions in mobile DHT systems," 12th International Symposium on Programming and Systems (ISPS), Algiers, Algeria, Apr. 2015, pp. 1-7, doi: 10.1109/ISPS.2015.7244963.

[6] J. Rybicki, B. Scheuermann, M. Koegel, and M. Mauve, (2009) "PeerTIS: a peer-to-peer traffic information system," in Proceedings of the sixth ACM international workshop on VehiculAr InterNETworking - VANET '09, Beijing, China, p. 23, doi: 10.1145/1614269.1614275.

[7] Q. Le-Dang, J. McManis, and G.-M. Muntean, (2014) "Location-Aware Chord-Based Overlay for Wireless Mesh Networks," IEEE Trans. Veh. Technol., vol. 63, no. 3, pp. 1378-1387, doi: 10.1109/TVT.2013.2284793.

[8] S. G. Fantar and H. Youssef, (2009) "Locality-aware Chord over Mobile Ad Hoc Networks," in 2009 Global Information Infrastructure Symposium, Hammemet, Tunisia, pp. 1-6, doi: 10.1109/GIIS.2009.5307057.

[9] Santos R. A., Edwards R. M., (2004) "Supporting inter-vehicular and vehicle roadside communications over a cluster-based wireless ad-hoc routing algorithm", Proc. International Symposium on Information and Communication Technologies(WISICT'04), Trinity College, Dublin, pp. 1-6.

[10] Y. Gunter, B. Wiegel, and H. P. Grossmann, (2007) "Medium Access Concept for VANETs Based on Clustering," IEEE 66th Vehicular Technology Conference, Baltimore, MD, pp. 2189-2193, doi: 10.1109/VETECF.2007.459.

[11] O. Kayis and T. Acarman, (2007) "Clustering Formation for Inter-Vehicle Communication," in 2007 IEEE Intelligent Transportation Systems Conference, Bellevue, WA, USA, pp. 636-641, doi: 10.1109/ITSC.2007.4357779.

[12] M. Akila and T. Iswarya, (2011)“An efficient data replication method for data access applications in vehicular ad-hoc networks," International Conference on Electronics, Communication and Computing Technologies, Villupuram District, Tamilnadu, India, Sep. 2011, pp. 17-22, doi: 10.1109/ICECCT.2011.6077062.

[13] S. Park and S. Lee, (2012) "Improving Data Accessibility in Vehicle Ad hoc Network," International Journal of Smart Home, vol. 6, no. 4, p. 8.

[14] M. H. Ghavifekr and A. C. Khosrowshahi, (2015) "Parking based Data Replication in VANET," Indian Journal of Science and Technology, vol. 8, no. 27, doi: 10.17485/ijst/2015/v8i27/70054.

[15] X. Fan, C. Huang, J. Zhu, and B. Fu, (2018) "R-DRA: a replication-based distributed randomized algorithm for data dissemination in connected vehicular networks," Wireless Networks, vol. 25, no. 7, pp. 3767-3782, doi: 10.1007/s11276-018-01895-3.

[16] Y. Wu, Y. Zhu, H. Zhu, and B. Li, (2013) "CCR: Capacity-constrained replication for data delivery in vehicular networks," in 2013 Proceedings IEEE INFOCOM, Turin, Italy, pp. 2580-2588, doi: 10.1109/INFCOM.2013.6567065. 
International Journal of Computer Networks \& Communications (IJCNC) Vol.12, No.6, November 2020

[17] J. Zhu, C. Huang, X. Fan, S. Guo, and B. Fu, (2018) "EDDA: An Efficient Distributed Data Replication Algorithm in VANETs," Sensors, vol. 18, no. 2, p. 547, doi: 10.3390/s18020547.

[18] J. B. Kenney, (2011) "Dedicated Short-Range Communications (DSRC) Standards in the United States," Proc. IEEE, vol. 99, no. 7, pp. 1162-1182, doi: 10.1109/JPROC.2011.2132790.

[19] S. Ratnasamy, P. Francis, M. Handley, S. Shenker, and R. Karp, (2001) "A Scalable ContentAddressable Network,” Proc. SIGCOMM’01, San Diego, California, USA, pp. 161-172.

[20] http://www.oversim.org/

[21] http://www.omnetpp.org/

[22] A. Wegener, M. Piórkowski, M. Raya, H. Hellbrück, S. Fischer, and J.-P. Hubaux, (2008) “TraCI: an interface for coupling road traffic and network simulators," in Proceedings of the 11th communications and networking simulation symposium on - CNS '08, Ottawa, Canada, p. 155, doi: 10.1145/1400713.1400740.

[23] C. Sommer, Zheng Yao, R. German, and F. Dressler, (2008) "Simulating the influence of IVC on road traffic using bidirectionally coupled simulators," in IEEE INFOCOM Workshops 2008, Phoenix, AZ, pp. 1-6, doi: 10.1109/INFOCOM.2008.4544655.

[24] F. K. Karnadi, Z. H. Mo, and K. Lan, (2007)“Rapid Generation of Realistic Mobility Models for VANET," in 2007 IEEE Wireless Communications and Networking Conference, Kowloon, China, pp. 2506-2511, doi: 10.1109/WCNC.2007.467.

[25] D. Krajzewicz, G. Hertkorn, P. Wagner, and C. Rössel, (2002) "SUMO (Simulation of Urban MObility) An open-source traffic simulation," Proc. Fourth Middle East Symposium. Simulation and Modelling (MESM '02), Sharjah, United Arab Emirates pp. 183-187. 


\section{Authors}

Abdelkader Guezzi - received his BS and MSc degrees from Kasdi Marbah University, Algeria, in 2009 and 2011, respectively. Now, he is a PhD student at the Department of Computer Science and Information Technology, Kasdi Marbah University. His research interests include computer networks, wireless and mobile networks.

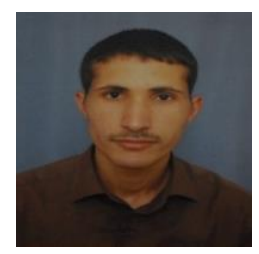

Abderrahmane Lakas - received the M.Sc. and Ph.D. degrees in computer systems from the University of Paris 6, France, in 1996. He is with the Department of Computer and Network Engineering, United Arab Emirates University, United Arab Emirates, since 2003. His research interests are in the areas of wireless and mobile networks, quality of service, vehicular ad hoc networks, connected autonomous systems, unmanned aerial vehicles, and the Internet of Things. He is currently leading the CAST (Connected Autonomous Intelligent Systems) Research Laboratory. Before joining United Arab Emirates University, he has held technical positions in few high-tech companies in Canada and USA, such as Newbridge Networks, Ottawa, Canada, from 1996 to 1998, Nortel Networks, Ottawa, from 1998 to 2000, and Netrake, Dallas, TX, USA, from 2000 to 2002

Ahmed Korichi - received his engineer degree in Computer Science from the University of Batna (Algeria) in 1987 and his Master's and $\mathrm{PhD}$ degrees in Computer Science from the University of Batna, Algeria in 2001 and 2009, respectively. He obtained his "HDR" degree from the University of Biskra, Algeria in 2010. He joined the Department of Computer Science and Information Technology at the University of Ouargla, Algeria. Since 2001, Dr. Ahmed Korichi has been research in the areas of network design and performance, modelling and simulation, QoS, wireless ad hoc networks, and vehicular networks. He has reviewed contributions in several scientific journals.

Sarra Cherbal - received her master's degree in networks and distributed systems in 2012 from Setif University (Algeria). Now she is a PhD student in Bordj Bou Arrerij University (Algeria). Her research interests include structured peer-to-peer systems on fixed and mobile networks. 